\section{AB1314-HPR ECONOMIC EVALUATION OF THE DR. BART APP IN PEOPLE WITH KNEE AND/OR HIP OSTEOARTHRITIS}

T. Pelle ${ }^{1,2}$, K. Bevers ${ }^{1}$, F. Van den Hoogen ${ }^{1,2}$, J. Van der Palen ${ }^{3}$, C. Van den Ende ${ }^{1,2} .{ }^{1}$ Sint Maartenskliniek, Nijmegen, Netherlands; ${ }^{2}$ Radboudumc, Nijmegen, Netherlands; ${ }^{3}$ University of Twente, Enschede, Netherlands

Background: Self-management is of paramount importance in non-surgical treatment of knee and/or hip osteoarthritis (OA). Modern technologies offer the possibility to support self-management $24 / 7$. We developed an e-self-management application (dr. Bart app) for people with knee and/or hip OA ${ }^{1}$.

Objectives: To evaluate the (incremental) cost-utility and cost-effectiveness of the dr. Bart app compared to usual care in people with knee/hip OA, applying a health care perspective.

Methods: This economic evaluation was conducted alongside a 6-month randomized controlled trial, in which 214 participants were offered to use the dr. Bart app for 6 months and 213 participants received care as usual. Health care costs were measured using self-reported questionnaires. Clinical outcome measures were quality-adjusted life years (QALYs) according to the EuroQol (EQ-5D-3L), the EuroQol rating scale (QALY-TRS), and the five subscales of KOOS/HOOS. Cost and effect differences were estimated using longitudinal linear mixed models and cost-effectiveness acceptability curves. Bootstrapping was used to estimate statistical uncertainty. Results: Mean age of participants was 62.1 (SD 7.3) years, with the majority being female (72\%) (Table 1). The difference in health care costs was non-significantly in favour of the intervention group ( $€-31.12(95 \% \mathrm{Cl}:-66 ; 3))$. Table 2 shows estimated treatment effects over 6 months. We found small but positive effects on symptoms, pain and activities of daily living (ADL) in favour of the dr. Bart app group. For QALY and QALY-TRS, the probability of the dr. Bart app being cost-effective compared to usual care was 0.80 and 0.60 at a willingness to pay (WTP) of $€ 10.000$ and 0.72 and 0.44 at WTP $€ 80.000$, respectively. For symptoms, pain and ADL, the probability that dr. Bart app was cost-effective was $>82 \%$ and for activities and quality of life $<40 \%$, regardless of WTPs.

Table 1. Baseline characteristics.

\begin{tabular}{lcc}
\hline & Dr. Bart app group $(\mathrm{n}=\mathbf{2 1 4})$ & Control group $(\mathrm{n}=\mathbf{2 1 3})$ \\
\hline Age, years; mean (SD) & $62.1(7.7)$ & $62.1(7.0)$ \\
Female, $\mathrm{n}(\%)$ & $147(68.7)$ & $159(74.7)$ \\
BMI, kg/m $\mathbf{m}^{2}$ mean (SD) & $27.8(5.1)$ & $27.3(4.8)$ \\
Main OA-location, knee; $\mathbf{n ( \% )}$ & $157(73.4)$ & $156(73.2)$ \\
\hline
\end{tabular}

Conclusion: This economic evaluation, from a health care perspective, showed that costs were not significantly lower for the dr. Bart app group compared to usual care. Given the non-invasive character of the intervention and the moderate probability to be cost-effective for the majority of outcomes, the dr. Bart app has only the potential to serve as a trustworthy tool to provide education and goal setting regarding $O A$ and its treatment options.

Table 2. Estimated treatment effects over 6 months.

\begin{tabular}{lccc}
\hline & $\begin{array}{c}\text { Estimated difference over } \mathbf{6} \text { months } \\
\text { between groups } \mathbf{( 9 5 \% ~ C l )}\end{array}$ & $\begin{array}{c}\text { WTP } \\
€ 10,000\end{array}$ & $\begin{array}{c}\text { WTP } \\
€ 80,000\end{array}$ \\
\hline Health care costs, $€$ & $-31(-66 ; 3)$ & $\mathrm{N} / \mathrm{A}$ & $\mathrm{N} / \mathrm{A}$ \\
QALY & $0.0045(-0.0119 ; 0.0210)$ & 0.80 & 0.72 \\
QALY-TRS & $-0.0015(-0.0149 ; 0.0111)$ & 0.60 & 0.44 \\
KOOS/HOOS & $2.6(-0.8 ; 5.8)$ & 0.93 & 0.93 \\
- Symptoms & $3.0(-0.2 ; 6.1)$ & 0.97 & 0.97 \\
- Pain & $1.9(2.3 ; 6.1)$ & 0.82 & 0.82 \\
- ADL & $-0.7(-5.1 ; 3.8)$ & 0.37 & 0.37 \\
- Activities & $-0.7(-4.8 ; 3.2)$ & 0.36 & 0.36 \\
- Quality of life & & &
\end{tabular}

${ }^{*}$ Control group serving as reference category. Abbreviations: KOOS; Knee Injury and Osteoarthritis Outcome Score; HOOS; Hip Disability and Osteoarthritis Outcome Score.

\section{References:}

[1] Pelle T, Bevers K, van der Palen J, van den Hoogen FHJ, van den Ende $\mathrm{CHM}$. Development and evaluation of a tailored e-self-management intervention (dr. Bart app) for knee and/or hip osteoarthritis: study protocol. BMC Musculoskelet Disord [Internet]. 2019 Dec 31;20(1):398.

Disclosure of Interests: None declared

DOI: 10.1136/annrheumdis-2020-eular.1721

\section{AB1315-HPR HYPERBARIC OXYGEN THERAPY IN FIBROMYALGIA PATIENTS - DOUBLE-BLIND PROSPECTIVE CLINICAL TRIAL}

B. Raffeiner ${ }^{1}$, F. Ometto ${ }^{2,3}$, D. Astorri ${ }^{3}$, C. Botsios ${ }^{3}$, G. Pulga ${ }^{4} .{ }^{1}$ Bolzano Hospital, Medicine Department, Bolzano, Italy; ${ }^{2}$ Local Health Unit 1 , Medicine
Department, Belluno, Italy; ${ }^{3}$ University of Padova, System's Department, Padova, Italy; ${ }^{4}$ Bolzano Hospital, Hyperbaric Unit, Bolzano, Italy

Background: Hyperbaric Oxygen Therapy (HOT) proved effective in improving of symptoms of patients affected by fibromyalgia syndrome (FMS) [1].

Objectives: The objective of the present study was to evaluate the effectiveness of HOT compared to hyperbaric treatment with no oxygen therapy (PBO) in the symptoms and working ability in FMS.

Methods: We conducted a prospective trial in employed patients with FMS, randomly assigned to HOT or PBO. Patients and evaluating clinicians were blinded to the treatment. HOT arm comprised 40 sessions, 5 days/week, 120 minutes, $100 \%$ oxygen at 2ATA; PBO comprised the same sessions without oxygen. Evaluations were at baseline, after 4 (T1) and 8 weeks (T2). Parameters considered were: socio-demographics, biochemistry, clinical evaluation and patient-reported outcomes (PROs). Baseline assessment included questions BELIEF (how much do you hope to improve with this treatment) and HOPE (how much do you expect to improve with this treatment), with VAS response. Spearman's, Mann-Whitney's, Kruskal-Wallis and Fisher's Exact test were used.

Results: 12 patients were included and completed the study, 6 in each arm (Tab. 1). No significant difference was observed in clinical measures or PROs at $\mathrm{T} 1$ and T2 between HOT and PBO arms, except for Working Productivity and Activity Impairment Questionnaire (WPAI) (result III) (Tab. 2). In both arms, disease duration was associated with worse PROs (Widespread pain index $r=0.59, p=0.037$, Severity Score $r=0.81, p=0.025$ ); higher BMI with improvement in function at $\mathrm{T} 2(\mathrm{r}=0.63, \mathrm{p}=0.027)$; higher baseline scores in $\mathrm{BELIEF}$ with reduction symptoms number $(r=-0.67, p=0.021)$, higher scores in HOPE with reduction in Health Assessment Questionnaire $(r=-0.057, p=0.039)$

Table 1. Patients characteristics

\begin{tabular}{cccc}
\hline & All & HOT & PBO \\
\hline Number & 12 & 6 & 6 \\
Age* $^{*}$ & $55,5(44 ; 59,75)$ & $55,5(47,75 ; 60)$ & $51(41 ; 58,75)$ \\
Females $^{* *}$ & $6(100)$ & $6(100)$ & $6(100)$ \\
Disease duration $^{*}$ & $10(8,25 ; 26,75)$ & $9,5(7,5 ; 20,75)$ & $15(10 ; 26,75)$ \\
BMl $^{*}$ & $25,5(22,25 ; 31)$ & $25(22,75 ; 28)$ & $28,5(23 ; 31,75)$ \\
Smoke $^{* *}$ & $0,5(0 ; 1)$ & $0(0 ; 1)$ & $1(0,25 ; 1)$ \\
HOPE score* $^{*}$ & $0(0 ; 1)$ & $0,5(0 ; 1)$ & $0(0 ; 0)$ \\
>=80** & $9(64,3)$ & $5(62,5)$ & $4(66,7)$ \\
BELIEF score* & $70(60 ; 80)$ & $75(67,5 ; 83,75)$ & $62,5(60 ; 68,75)$ \\
$>==70^{* *}$ & $8(57,1)$ & $6(75)$ & $2(33,3)$ \\
\hline
\end{tabular}

${ }^{*}$ median (IQR); ${ }^{* *}$ number (\%)

Table 2. Change from baseline in clinical measures and PROs at T2.

\begin{tabular}{|c|c|c|c|c|c|c|c|c|}
\hline & \multicolumn{3}{|c|}{ Medians (IQR) } & \multirow[t]{2}{*}{$\begin{array}{c}\mathbf{P} \\
\text { value }\end{array}$} & \multicolumn{3}{|c|}{$\begin{array}{c}\geqq 20 \text { Percentage } \\
\text { amelioration No. } \\
(\%)\end{array}$} & \multirow[t]{2}{*}{$P$ value } \\
\hline & All & нОт & PBO & & All & нОТ & PBO & \\
\hline $\begin{array}{l}\text { Short Form-36 } \\
\text { - Physical }\end{array}$ & $1(-1 ; 4)$ & $3(-1 ; 5,5)$ & $0,5(-0,75 ; 3,25)$ & ns & $0(0)$ & $0(0)$ & $0(0)$ & ns \\
\hline SF-36 - Mental & $4(-1 ; 8)$ & $6(2,5 ; 9,5)$ & $1(-1,75 ; 5,25)$ & ns & $0(0)$ & $0(0)$ & $0(0)$ & ns \\
\hline $\begin{array}{l}\text { Severity score } \\
\text { - total }\end{array}$ & $-13(-15 ;-2)$ & $\begin{array}{c}-15 \\
(-17 ;-13,5)\end{array}$ & $-3(-5,5 ;-0,5)$ & ns & $2(18,2)$ & $2(40)$ & $0(0)$ & ns \\
\hline Number symptoms & $-3(-4 ; 0)$ & $\begin{array}{c}-4 \\
(-4,5 ;-1,5)\end{array}$ & $-2(-3 ;-0,25)$ & ns & $2(18,2)$ & $2(40)$ & $0(0)$ & ns \\
\hline SASP score & $-1(-3 ; 0)$ & $\begin{array}{c}-2 \\
(-3,5 ;-0,5)\end{array}$ & $-1(-1,75 ;-0,25)$ & ns & $1(9,1)$ & $0(0)$ & $\begin{array}{c}1 \\
(16,7)\end{array}$ & ns \\
\hline $\begin{array}{l}\text { Widespread pain } \\
\text { index }\end{array}$ & $0(-2 ; 0)$ & $0(-2 ; 0)$ & $-0,5(-1,75 ; 0)$ & ns & $2(18,2)$ & $2(40)$ & $0(0)$ & ns \\
\hline $\begin{array}{l}\text { Tender Points } \\
\text { Count }(0-18\end{array}$ & $-2(-3 ;-0,5)$ & $-2(-3,5 ;-1)$ & )$-1,25(-2 ;-0,13)$ & ns & $3(27,3)$ & $2(40)$ & $\begin{array}{c}1 \\
(16,7)\end{array}$ & Ns \\
\hline Health Assessment & t $0(0 ; 0)$ & 0 & $0(0 ; 0)$ & ns & $3(27,3)$ & $1(20)$ & 2 & ns \\
\hline $\begin{array}{l}\text { Questionnaire } \\
\text { Fibromyalgia } \\
\text { Impact } \\
\text { Questionnaire }\end{array}$ & $10(0 ; 15)$ & $\begin{array}{l}(-0,5 ; 0,5) \\
10(7,5 ; 15)\end{array}$ & $2,5(-3,75 ; 12,5)$ & ns & $1(9,1)$ & $0(0)$ & $\begin{array}{c}(33,3) \\
1 \\
(16,7)\end{array}$ & ns \\
\hline WPAI result-2 & $\begin{array}{c}-0,5 \\
(-1,25 ;-0,13)\end{array}$ & $\begin{array}{c}-1,5 \\
(-2 ;-0,5)\end{array}$ & $\begin{array}{c}-0,25 \\
(-0,5 ; 0,19)\end{array}$ & ns & $3(21,4)$ & $0(0)$ & $\begin{array}{c}3 \\
(37,5)\end{array}$ & ns \\
\hline WPAI result-3 & $0(-2,38 ; 0)$ & $\begin{array}{c}-2,75 \\
(-3,88 ;-2)\end{array}$ & $0(0 ; 0)$ & ns 5 & $5(35,7)$ & $0(0)$ & $\begin{array}{c}5 \\
(62,5)\end{array}$ & $P=0.008$ \\
\hline WPAI result-1 & $\begin{array}{c}-1(-3,25 ;- \\
0,38)\end{array}$ & $\begin{array}{c}-3,5 \\
(-4,75 ;-1,5)\end{array}$ & $-0,5(-1 ; 0,38)$ & ns & $6(42,9)$ & $\begin{array}{c}2 \\
(33,3)\end{array}$ & $4(50)$ & ns \\
\hline
\end{tabular}

Conclusion: 8-week HOT treatment does not substantially improve symptoms in FMS compared to PBO. All patients on hyperbaric treatment may experience amelioration of symptoms: other factors should be considered, including beliefs and expectations on the treatment. 